\title{
Aplicação de água salina no desenvolvimento e comportamento fisiológico do coqueiro
}

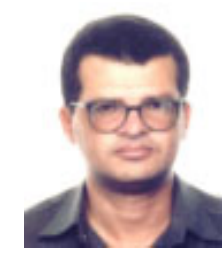

Carlos D. da Silva Júnior ${ }^{1}$, Edson E. M. Passos² \& Hans R. Gheyi ${ }^{3}$

\footnotetext{
1 Depto. de Biologia da Universidade Federal de Sergipe, CEP 49100-000, São Cristóvão, SE. E-mail: cdias@ufs.br (Foto)

2 Embrapa Tabuleiros Costeiros. CP 44, CEP 49001-970, Aracaju, SE. E-mail: edson@cpatc.embrapa.br

3 DEAg/CCT/UFPB. Av. Aprígio Veloso, 882, Bodocongó, CEP 58109-970, Campina Grande, PB. Fone: (83) $310-1056$. E-mail: hans@deag.ufpb.br
}

Protocolo 139 - 27/9/2001

\begin{abstract}
Resumo: Avaliou-se o efeito do déficit hídrico e da irrigação com água de diferentes níveis de salinidade, no desenvolvimento do coqueiro (Cocos nucifera L.) "Gigante do Brasil", com cinco anos de idade e cultivado em condições de campo. O experimento foi conduzido no Campo Experimental da Embrapa Tabuleiros Costeiros, localizado no município de Itaporanga D'Ajuda, SE. O delineamento experimental utilizado foi em blocos ao acaso, com quatro tratamentos e quatro repetições. Cada parcela foi constituída de 12 plantas úteis. Os tratamentos foram: plantas sem irrigação $\left(T_{0}\right)$, aplicação de $40 \mathrm{~L}$ de água doce por planta em intervalos de 3 dias $\left(T_{1}\right)$, aplicação de água contendo 7,5 e $15,0 \mathrm{~g} \mathrm{~L}^{-1}$ de sais totais $\left(T_{2}\right.$ e $\left.T_{3}\right)$. Analisaram-se as seguintes variáveis: número de folhas vivas, número de folhas mortas, porcentagem de plantas vivas no final do experimento, potencial hídrico foliar e condutância estomática. A análise dos dados demonstrou que os tratamentos $T_{0}$ e $T_{3}$ diferiram significativamente quanto ao número de folhas vivas, mas a variável condutância estomática no período seco foi afetada em todos os tratamentos, o que mostrou a insuficiência da quantidade de água aplicada. No final do experimento, a percentagem de sobrevivência foi de $83 \%$ para $T_{1}, 81 \%$ para $T_{2}, 79 \%$ para $T_{3}$ e $58 \%$ para $T_{0}$. Conclui-se, então, que a irrigação com água contendo até 15 $\mathrm{g} \mathrm{L}^{-1}$ de sais em períodos de seca transitória, é essencial para evitar a perda de plantas jovens do coqueiro "Gigante do Brasil", no Nordeste do Brasil.
\end{abstract}

Palavras-chave: Cocos nucifera L., salinidade, condutância estomática, déficit hídrico, relações hídricas

\section{Application of saline water in the development and physiological behavior of the coconut}

\begin{abstract}
This work was carried out with the objective of evaluating the effect of the drought and the application of water of different levels of salinity in the development of five years old "Brazilian Tall" coconut (Cocos nucifera L.) cultivated under field conditions. The experiment was conducted at the Itaporanga Experimental Station in the State of Sergipe located in Northeast of Brazil. The experimental design consisted of randomized blocks (4) with 4 treatments and each repetition constituted of 12 plants. The applied treatments were: $T_{0}$ - not irrigated; $T_{1}$ - application of $40 \mathrm{~L}$ per plant of fresh water at intervals of 3 days; $T_{2}$ and $T_{3}$ - application of saline water containing 7.5 and $15.0 \mathrm{~g} \mathrm{~L}^{-1}$ of total salts. The following variables were analyzed: number of living leaves, number of dead leaves, the percentage survival of plants at the end of the experiment, the leaf water potential and the stomatal conductance. The analysis of the data showed that the treatments $\mathrm{T}_{0}$ and $\mathrm{T}_{3}$ affected significantly the number of living leaves, but the variable stomatal conductance in the dry period was affected by all the treatments, which points out the inadequacy of the amount of water applied. At the end of the experiment, the survival percentage was of $83 \%$ for $T_{1}, 81 \%$ for $T_{2}, 79 \%$ for $T_{3}$ and $58 \%$ for $T_{0}$. The results permit to conclude that water containing up to $15 \mathrm{~g} \mathrm{~L}^{-1}$ salts during dry season is essential to avoid the loss of young plants.
\end{abstract}

Key words: Cocos nucifera L., salinity, stomatal conductance, water stress, water relations

\section{INTRODUÇÃO}

A salinização é um dos principais fatores que afetam milhões de hectares de terra ao redor do mundo, devido ao desmatamento, ao uso intensivo para à agricultura e à irrigação excessiva.
Extensas áreas apresentam-se bastante prejudicadas pela salinidade, principalmente na África, sudoeste da Ásia, Austrália e América Central (Dudal \& Purnell, 1986; Ghassemi et al., 1995).

As plantas reagem a salinidade ou o estresse hídrico, reduzindo seu crescimento, devido à redução do potencial hídrico e do 
potencial osmótico (Munns \& Termaat, 1986; Lin \& Sternberg, 1993) sendo essas reduções dos potenciais provenientes da dissolução dos sais (Ziska et al., 1989; Alarcón et al., 1993). Para Coudret \& Louguet (1980) o regime hídrico das plantas tolerantes ao $\mathrm{NaCl}$ nãoé modificado, como o que acontece com as plantas sensíveis.

A resistência das plantas aos sais varia em função do mecanismo adotado. A suculência, por exemplo, pode provocar aumento no teor de água (Boutelier, 1986) em comparação com a exclusão ou com a tolerância aos sais que podem provocar efeito contrário (Coudret, 1981). No caso do coqueiro, o estudo dos efeitos dos sais nas características hídricas e fisiológicas poderá fornecer informações complementares com relação à tolerância desta espécie à salinidade do solo como, também, verificar a possibilidade de se utilizar água salina na irrigação.

$\mathrm{O}$ efeito da salinidade em numerosas plantas de interesse agronômico foi estudado por vários autores, como Grunberg \& Taleisnik (1991) e Bourgeais-Chaillou et al. (1992). Trabalho executado na Costa do Marfim, por Pomier \& Brunin (1974) em condições de campo num solo arenoso, mostrou a possibilidade de se irrigar o coqueiro com água salobra contendo até $15 \mathrm{~g} \mathrm{~L}^{-1}$, durante o período de estiagem. Em condições de enviveiramento, Remison \& Iremiren (1990) constataram que o número de folhas de plantas jovens de coqueiro que tinham recebido de 2 a $12 \mathrm{~g}$ de sais, aplicados no solo, não foi afetado, comparando-se ao tratamento testemunha.

A redução de crescimento das plantas que recebem irrigação com água salina em comparação com as testemunhas, pode ser causada pela toxidez dos íons, redução da absorção de água e alteração dos processos fisiológicos e bioquímicos com o aumento do estresse salino (Munns \& Termaat, 1986 e Lin \& Sternberg, 1993).

Como a zona costeira do Nordeste do Brasil apresenta certa reserva de água doce no lençol freático e grande disponibilidade de água salina, do mar ou salobra nos rios, faz-se necessário conhecer a possibilidade da utilização dessas fontes de água para a irrigação durante os períodos mais secos, a fim de se limitar as conseqüências do déficit hídrico nessa cultura.

O objetivo do presente trabalho foi avaliar os efeitos da aplicação de água contendo diferentes teores de sais em plantas do coqueiro "Gigante do Brasil", fornecendo um melhor conhecimento das relações hídricas e da tolerância do coqueiro à salinidade, durante os períodos de déficit hídrico transitório.

\section{MATERIAL E MÉTODOS}

O experimento foi conduzido no Campo Experimental da Embrapa Tabuleiros Costeiros, localizado a $11^{\circ} 07^{\prime}$ de latitude Sul

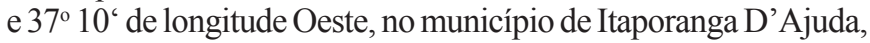
no estado de Sergipe. Foram utilizadas plantas da variedade Gigante do Brasil, com aproximadamente cinco anos de idade, no espaçamento de $9 \times 9 \times 9 \mathrm{~m}$ em triângulo, cultivadas em solo do tipo Areia Quartzosa distrófica, pobre em matéria orgânica $(0,25 \%)$. As adubações foram realizadas seguindo-se as recomendações de Sobral \& Santos (1987) com base na diagnose foliar. As doses de uréia e de cloreto de potássio foram fracionadas em duas aplicações anuais (início e final da estação chuvosa) e o superfosfato simples foi aplicado de uma só vez, no início da estação chuvosa.

A água foi aplicada por regador, em intervalo de três dias com 40 L por planta de água do mar diluída, de acordo com os tratamentos, nos meses em que a pluviosidade foi inferior a $150 \mathrm{~mm}$. A quantidade de água aplicada não corresponde ao que permitiria a manutenção da capacidade de campo; no entanto, ela foi determinada em função do volume de água diário que um pequeno produtor da região pode fornecer com os meios técnicos limitados. O delineamento experimental foi em blocos ao acaso, com quatro tratamentos, quatro repetições e 12 plantas úteis por parcela, Os tratamentos foram assim constituídos: testemunha - sem aplicação de água $\left(\mathrm{T}_{0}\right)$, aplicação de água doce contendo $0,1 \mathrm{~g} \mathrm{~L}^{-1}$ de sais totais $\left(\mathrm{T}_{1}\right)$, água contendo $7,5 \mathrm{~g} \mathrm{~L}^{-1}$ de sais totais $\left(\mathrm{T}_{2}\right)$ e $15 \mathrm{~g} \mathrm{~L}^{-1}$ de sais totais $\left(\mathrm{T}_{3}\right)$.

A pluviometria foi medida no campo experimental e a evapotranspiração potencial estimada pelo método de Hargreaves (1974) a partir das médias mensais de temperatura e da umidade relativa do ar, obtidas na estação meteorológica, localizada a $25 \mathrm{~km}$ do campo experimental. A profundidade do lençol freático foi monitorada mensalmente, em cada bloco.

Para se avaliar a intensidade e os efeitos do estresse na fenologia das plantas, utilizou-se as seguintes variáveis: número de folhas vivas, número de folhas mortas e porcentagem de plantas vivas no final do experimento.

As respostas fisiológicas das plantas foram avaliadas do potencial hídrico foliar e da condutância estomática, em um folíolo do terço médio da folha 8 a partir do ápice, em três plantas de cada tratamento, entre as 9 e as $10 \mathrm{~h}$ da manhã, em dois dias consecutivos (dois blocos no primeiro dia e dois blocos no segundo dia) durante o período de 14 meses. A condutância estomática foi medida com um porômetro a difusão de vapor de água (Delta T MK3-Device, Cambridge, UK). O potencial hídrico foliar foi medido no campo, utilizando-se de uma câmara de pressão (Scholander et al., 1965). Para se verificar o comportamento das plantas em diferentes estações, avaliou-se a evolução diária dos parâmetros fisiológicos durante a estação seca e a chuvosa.

A diagnose foliar foi realizada em doze plantas de cada tratamento, após sete meses. As folhas coletadas em cada tratamento foram desidratadas em estufa a $65^{\circ} \mathrm{C}$ durante $48 \mathrm{~h}$ e encaminhadas ao Laboratório de Fertilidade da Embrapa Tabuleiros Costeiros, para análise, segundo metodologia recomendada por Malavolta (1989).

Os resultados foram submetidos a análise de variância e as médias comparadas pelo teste de Tukey, a $5 \%$ de probabilidade.

\section{RESULTADOS E DISCUSSÃO}

A Figura 1 representa a distribuição mensal por decêndio, da quantidade de chuvas, mostrando a grande irregularidade da pluviometria durante o período experimental. Nos três últimos anos

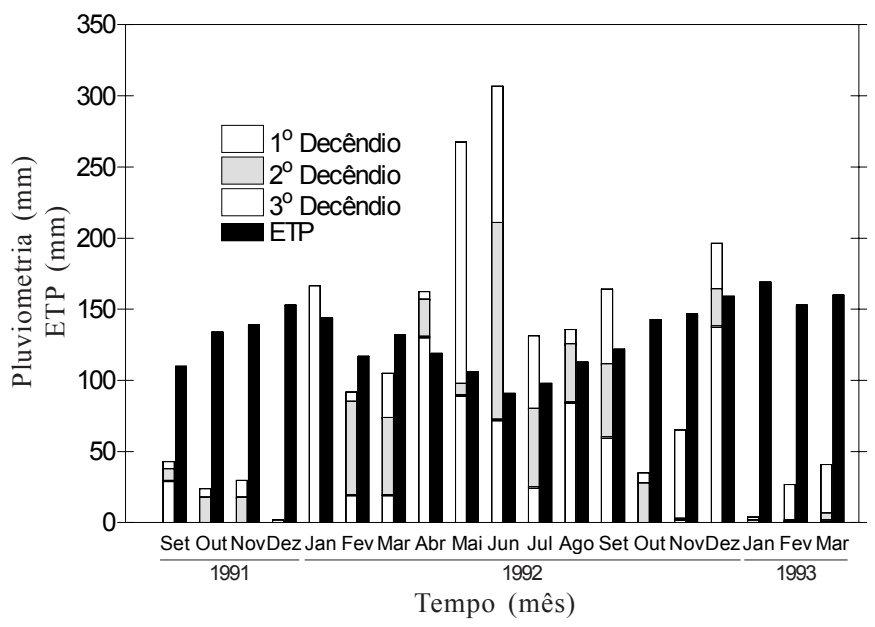

Figura 1. Evolução mensal da pluviosidade e da evapotranspiração potencial (ETP) durante o período de estudo, no Campo Experimental de Itaporanga, Sergipe, Brasil 
que antecederam o experimento, a pluviometria média anual foi em torno de $1800 \mathrm{~mm}$, o que fica evidente que, nessa região, o principal problema que interferiu no desenvolvimento das plantas estudadas não foi a quantidade total de chuvas, mas a distribuição irregular da pluviometria, além da fraca retenção de água por parte do solo. Durante o período de pluviometria reduzida e elevada evapotranspiração potencial, o déficit hídrico pode chegar a $434 \mathrm{~mm}$, como ocorreu entre os meses de setembro a dezembro de 1991.

Ficou evidente que o período de déficit hídrico e o acúmulo de sais no solo, este confirmado pelo acúmulo de íons $\mathrm{Na}^{+} \mathrm{e}$ $\mathrm{Cl}^{-}$nas folhas (Tabela 1) impuseram sérias restrições ao crescimento das plantas dos tratamentos que receberam água salina, causando, inclusive, morte de plantas (Figs. 2 3). No
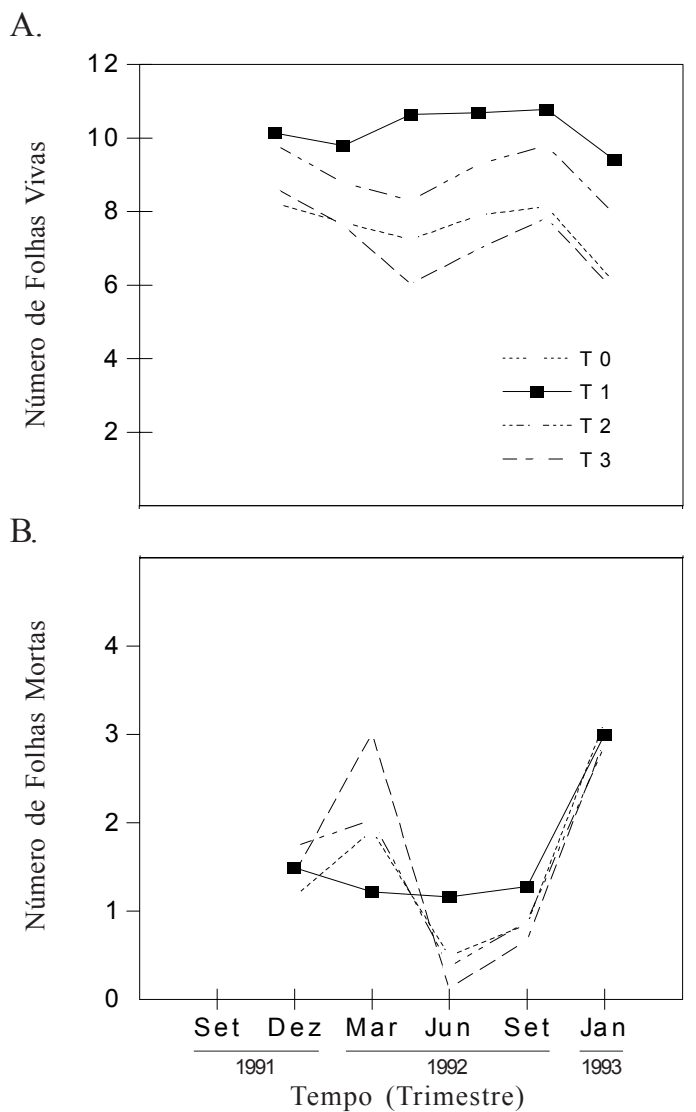

Figura 2. Evolução do número de folhas vivas $(\mathrm{A})$ e do número de folhas mortas (B) das plantas do coqueiro "Gigante do Brasil", sob diferentes tratamentos durante o período de setembro de 1991 a janeiro de 1993. $\mathrm{T}_{0}$ - testemunha, $\mathrm{T}_{1}$ - aplicação de água doce, $\mathrm{T}_{2}$ e $\mathrm{T}_{3}$ - aplicação de água contendo 7,5 e $15 \mathrm{~g} \mathrm{~L}^{-1}$ de sais totais, respectivamente

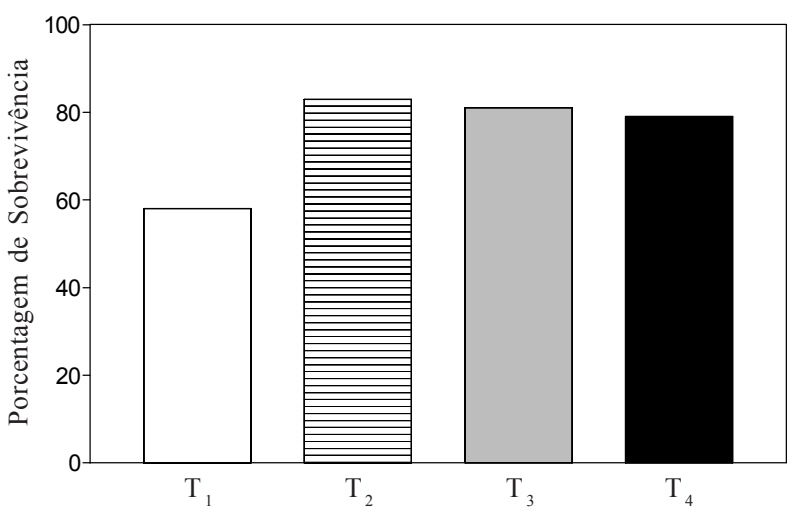

Figura 3. Porcentagem de sobrevivência das plantas do coqueiro "Gigante do Brasil", sob diferentes concentrações de sais em janeiro de 1993. $T_{0}$ - testemunha, $T_{1}$ - aplicação de água doce, $\mathrm{T}_{2}$ - água com 7,5 $\mathrm{g} \mathrm{L}^{-1}$ de sais dissolvidos totais e $\mathrm{T}_{3}$ - água com $15 \mathrm{~g} \mathrm{~L}^{-1}$ de sais totais

tratamento $\mathrm{T}_{1}$, durante todo o período de experimentação, o número de folhas vivas (Fig. 2A) foi superior aos demais tratamentos, com diferença significativa $(\mathrm{p}<0,01)$ com relação aos tratamentos $\mathrm{T}_{0}$ e $\mathrm{T}_{3}(\mathrm{p}<0,05)$ e comparado ao tratamento $\mathrm{T}_{2}$ as diferenças foram significativas a nível de 0,05 da probabilidade. No tratamento com 7,5 $\mathrm{g} \mathrm{L}^{-1}$ de sais totais $\left(\mathrm{T}_{2}\right)$ a diferença foi significativamente superior ao tratamento $T_{3}(p<0,01)$ e ao tratamento $T_{0}$ $(\mathrm{p}<0,05)$ ficando evidente a tolerância do coqueiro a esta concentração de sais na água. Remison \& Iremiren (1990) trabalhando com coqueiros jovens em viveiro, observaram que as plantas que receberam de 2 a $12 \mathrm{~g}$ de sal comum, não apresentaram diferença significativa quanto ao número de folhas vivas, comparadas com as plantas testemunha.

Uma das primeiras conseqüências do estresse hídrico e do acúmulo de sais no solo para o coqueiro Gigante do Brasil, foi uma redução da área foliar devido à perda de folhas (Fig. 2B). Nas plantas do tratamento $\mathrm{T}_{3}$ observou-se aspecto coriáceo das folhas; no entanto, após as chuvas, quando os sais foram lixiviados, as plantas retomaram o crescimento normal. A perda de folhas nas plantas irrigadas com água contendo $15 \mathrm{~g} \mathrm{~L}^{-1}$ de sais totais, pode ser atribuída à toxicidade de íons, redução da absorção de água e alteração dos processos fisiológicos e bioquímicos, com o aumento do estresse salino (Munns \& Termaat, 1986 e Lin \& Sternberg, 1993).

A taxa de sobrevivência (Fig. 3) das plantas na testemunha $\left(\mathrm{T}_{0}\right)$, foi inferior à dos demais tratamentos, pois a ausência de irrigação durante os períodos de deficiência hídrica induziu a alta taxa de mortalidade, apresentando apenas $58 \%$ das plantas vivas. Os tratamentos que receberam aplicação de água salina

Tabela 1. Composição mineral das folhas das plantas de coqueiro Gigante do Brasil com cinco anos de idade, sob diferentes tratamentos

\begin{tabular}{|c|c|c|c|c|c|c|c|}
\hline \multirow{2}{*}{ Tratamento $^{*}$} & \multicolumn{7}{|c|}{ Elementos Minerais na Folha (\% de Matéria Seca) ${ }^{* *}$} \\
\hline & $\mathrm{N}$ & $\mathrm{P}$ & $\mathrm{K}$ & $\mathrm{Ca}$ & $\mathrm{Mg}$ & $\mathrm{Cl}$ & $\mathrm{Na}$ \\
\hline $\mathrm{T}_{0}$ & $1,74 \mathrm{a}$ & $0,27 \mathrm{a}$ & $1,29 \mathrm{a}$ & $0,27 \mathrm{a}$ & $0,15 \mathrm{~b}$ & $0,40 \mathrm{c}$ & $0,14 \mathrm{~b}$ \\
\hline $\mathrm{T}_{2}$ & $1,59 \mathrm{a}$ & $0,19 \mathrm{~b}$ & $1,26 \mathrm{a}$ & $0,29 \mathrm{a}$ & $0,31 \mathrm{a}$ & $0,66 \mathrm{a}$ & $0,27 \mathrm{a}$ \\
\hline $\mathrm{T}_{3}$ & $1,71 \mathrm{a}$ & $0,19 \mathrm{~b}$ & $1,34 \mathrm{a}$ & $0,24 \mathrm{a}$ & $0,27 \mathrm{a}$ & $0,66 \mathrm{a}$ & $0,27 \mathrm{a}$ \\
\hline Média Geral & 1,69 & 0,22 & 1,35 & 0,27 & 0,22 & 0,56 & 0,21 \\
\hline
\end{tabular}

\footnotetext{
* $\mathrm{T}_{0}$ - Testemunha; $\mathrm{T}_{1}$ - Aplicação de água doce; $\mathrm{T}_{2}$ - Aplicação de água contendo $7,5 \mathrm{~g} \mathrm{~L}^{-1}$ de sais totais; $\mathrm{T}_{3}$ - Aplicação de água contendo $15 \mathrm{~g} \mathrm{~L}{ }^{-1}$ de sais totais
}

"* Os valores médios seguidos pela mesma letra na coluna não diferem significativamente pelo teste de Tukey, a 0,05 da probabilidade 
apresentaram taxa de sobrevivência de $81 \%\left(\mathrm{~T}_{2}\right)$ e $79 \%\left(\mathrm{~T}_{3}\right)$, respectivamente. No tratamento $\mathrm{T}_{1}$, o percentual de sobrevivência de $83 \%$, indicou que, embora o volume de água aplicada não tenha sido suficiente para compensar a ETP, permitiu a sobrevivência das plantas no período da baixa pluviosidade.

Resultados obtidos com diferentes espécies como, por exemplo, o sorgo (Bernstein et al., 1993a e b), o algodão (Alexander \& Woodham, 1968) e a lentilha (Katerji et al., 2001) mostram que existe um limite para cada cultura em que a concentração de $\mathrm{NaCl}$ pode estimular o crescimento, e que concentrações acima deste limite provocam redução importante da massa dos tecidos secos e da área foliar. Este limite de tolerância aos sais foi observado no presente trabalho, onde a aplicação de água contendo até $15 \mathrm{~g} \mathrm{~L}^{-1}$ de sais permitiu que o coqueiro mantivesse seus tecidos hidratados possibilitando, desta forma, manter a sua atividade metabólica, mesmo reduzida.

Os resultados dos teores em elementos minerais na folha são agrupados na Tabela 1 . Os teores de $\mathrm{P}, \mathrm{Mg}, \mathrm{Cl}$ e $\mathrm{Na}$ das plantas sob água salina $\left(\mathrm{T}_{2}\right.$ e $\left.\mathrm{T}_{3}\right)$ diferem dos obtidos nas plantas irrigadas com água doce $\left(\mathrm{T}_{1}\right)$. As plantas do tratamento $\mathrm{T}_{0}$ apresentaram teores de fósforo significativamente superiores aos tratamentos que receberam água salina $(\mathrm{p}<0,05)$, ficando evidente que o estresse salino dificultou a absorção desse elemento.

Nas plantas que receberam água salina ocorreu aumento significativo no teor de magnésio das folhas $(\mathrm{p}<0,05)$ em relação às plantas dos tratamentos $\mathrm{T}_{0}$ e $\mathrm{T}_{1}$. Os teores apresentados são da ordem de 94 e $69 \%$, respectivamente, mais elevados para os tratamentos $\mathrm{T}_{2}$ e $\mathrm{T}_{3}$.

Os teores de cloro e sódio na folha não diferem entre os tratamentos $\mathrm{T}_{2}$ e $\mathrm{T}_{3}$, sendo significativamente superiores $(\mathrm{p}<$ $0,05)$ aos tratamentos $\mathrm{T}_{0}$ e $\mathrm{T}_{1}$.

Os percentuais de sódio na folha são iguais para os tratamentos $\mathrm{T}_{2}$ e $\mathrm{T}_{3}$. As quantidades de sódio são mantidas aumentadas em $69 \%$, comparando-se ao tratamento $\mathrm{T}_{1}$.

A tolerância a salinidade em plantas glicófitas, está associada à habilidade para limitar o acúmulo e/ou o transporte de íons salinos, principalmente $\mathrm{Na}^{+}$e o $\mathrm{Cl}^{-}$, da zona da raiz para a parte aérea das plantas (Greenway \& Munns, 1980). A maior concentração em $\mathrm{Cl}^{-}$que de $\mathrm{Na}^{+}$na parte aérea, pode resultar das diferentes capacidades que essas plantas possuem de compartimentar esses íons nos vacúolos. Plantas consideradas não halófitas, parecem ser capazes de acumular $\mathrm{Cl}^{-}$; no entanto, muitas não dispõem de mecanismos para fazer a inclusão de $\mathrm{Na}^{+}$nos vacúolos (Mennen et al., 1990). No caso do coqueiro, essas plantas possuem capacidade de armazenar íons $\mathrm{Na}^{+} \mathrm{e} \mathrm{Cl}^{-}$ nas folhas, sendo que o $\mathrm{Cl}^{-}$em maior proporção.

A evolução do potencial hídrico das folhas durante o período de experimentação é mostrado na Figura 4. Durante os quatro meses de menor pluviosidade e maior aprofundamento do lençol freático, estando no mês de janeiro a $2,10 \mathrm{~m}$ da superfície do solo, as plantas sob tratamentos com água salina $\mathrm{T}_{2}$ e $\mathrm{T}_{3}$ e as do tratamento $\mathrm{T}_{0}$ sem aplicação, apresentaram redução do potencial hídrico da ordem de 20, 23 e 30\%, respectivamente, comparando-se com as plantas de $\mathrm{T}_{1}$. Após o final do mês de janeiro, o aumento da precipitação e a elevação do lençol freático, atingindo 1,0 $\mathrm{m}$ nos meses de maior pluviosidade, influenciaram nas respostas das plantas à salinidade. Em condições naturais, diferentes fatores, como a umidade do solo, as chuvas e o nível do lençol freático, variam continuamente, e as medidas obtidas resultam diretamente da influência desses diferentes fatores. As variações registradas não foram suficientes para evidenciar diferenças entre os tratamentos, nos quais não se constatou redução do potencial hídrico que seja diretamente ligada à concentração de sais; resultados similares foram encontrados por Boutelier (1986) em plantas de algodão, evidenciando que a redução do potencial hídrico não está diretamente correlacionada ao aumento da concentração de $\mathrm{NaCl}$ no meio de cultura.

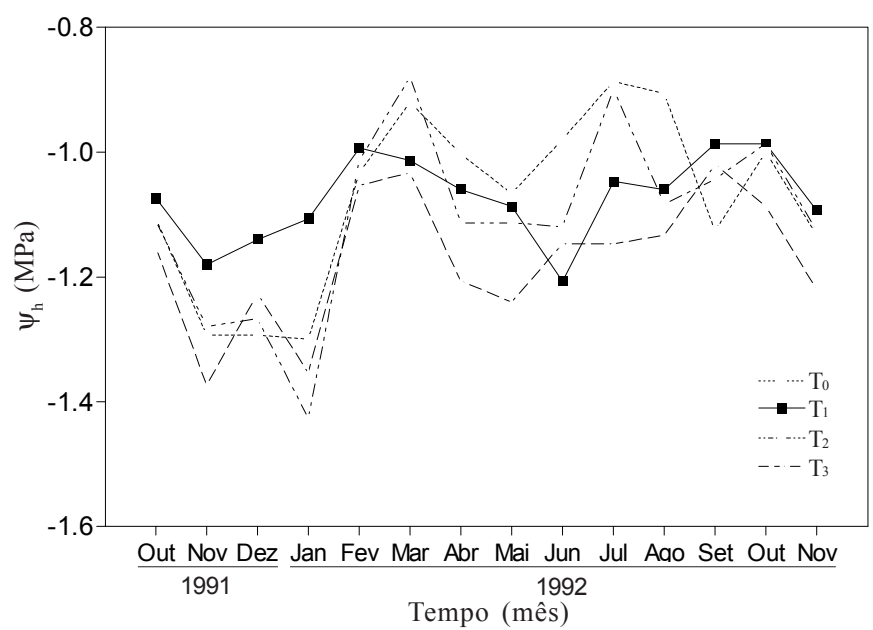

Figura 4. Evolução do potencial hídrico foliar $\Psi_{\mathrm{h}}(\mathrm{MPa})$ em função do tempo, em plantas do coqueiro "Gigante do Brasil", sob diferentes tratamentos durante outubro de 1991 a novembro de 1992. $\mathrm{T}_{0}$ - testemunha, $\mathrm{T}_{1}$ - aplicação de água doce, $\mathrm{T}_{2}$ e $\mathrm{T}_{3}$ - aplicação de água contendo 7,5 e $15 \mathrm{~g} \mathrm{~L}^{-1}$ de sais totais, respectivamente

A condutância estomática (gs) das plantas dos tratamentos $\mathrm{T}_{0}, \mathrm{~T}_{2}$ e $\mathrm{T}_{3}$ foi inferior ao tratamento com água doce $\left(\mathrm{T}_{1}\right)$, durante quase todo o período experimental (Fig. 5). A análise de variância das médias mostraram diferenças significativas apenas entre os tratamentos $\mathrm{T}_{1}$ e $\mathrm{T}_{0}$ a nível de 0,05 da probabilidade.

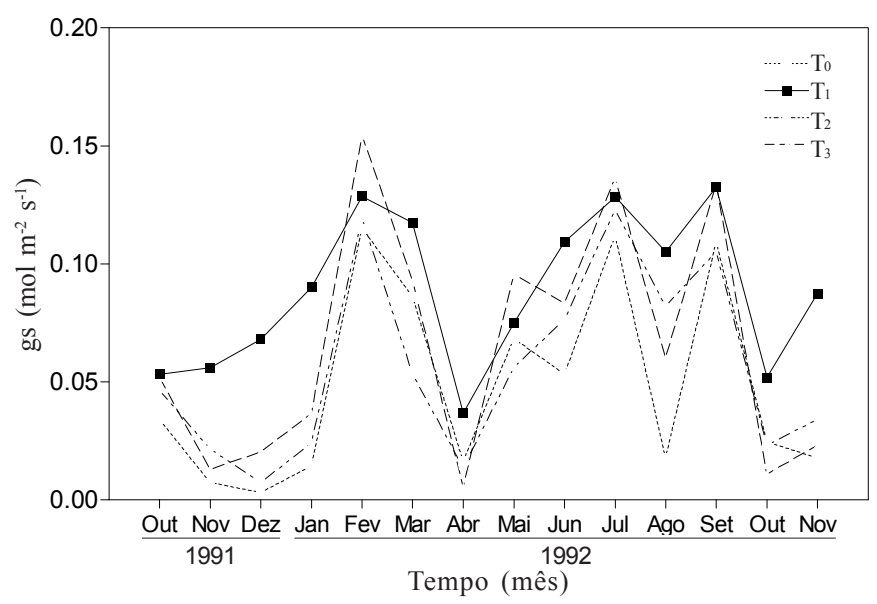

Figura 5. Evolução da condutância estomática - gs em função do tempo, em plantas do coqueiro "Gigante do Brasil", sob diferentes tratamentos de outubro de 1991 a novembro de 1992. $\mathrm{T}_{0}$ - testemunha, $\mathrm{T}_{1}$ - aplicação de água doce, $\mathrm{T}_{2}$ e $\mathrm{T}_{3}$ - aplicação de água contendo 7,5 e $15 \mathrm{~g} \mathrm{~L}^{-1}$ de sais totais, respectivamente 
Rozema \& van Diggelan (1991) demonstraram que, mesmo para algumas halófitas, quando os níveis de sais aplicados são elevados, podem causar redução da abertura estomática, induzindo a diminuição da transpiração e, conseqüentemente, da produção primária.

Como as condições climáticas variam durante o dia, em particular a temperatura, a umidade relativa do ar e a luminosidade, foi avaliada a evolução diária, do potencial hídrico e da condutância estomática, nas estações seca e chuvosa.

No que concerne ao período chuvoso (Fig. 6A) as plantas de todos os tratamentos apresentaram o potencial hídrico na ordem de $-0,8 \mathrm{MPa}$ às $8 \mathrm{~h}$, reduzindo muito pouco nas horas mais quentes do dia, sem apresentar diferença considerável entre os tratamentos.

Durante a estação seca (Fig. 6B) o potencial hídrico foliar nos tratamentos $\mathrm{T}_{0}$ e $\mathrm{T}_{3}$ foi inferior ao das plantas em $\mathrm{T}_{1} \mathrm{e} \mathrm{T}_{2}$. As plantas do tratamento $\mathrm{T}_{3}$ mostraram potencial hídrico significativamente inferior ao dos demais tratamentos $(\mathrm{p}<0,01)$. Deste modo, fica evidente que, durante a estação seca, concentrações mais elevadas de sais na água aplicada provocam maior desidratação do coqueiro que a ausência da irrigação. O baixo

A. Período Chuvoso

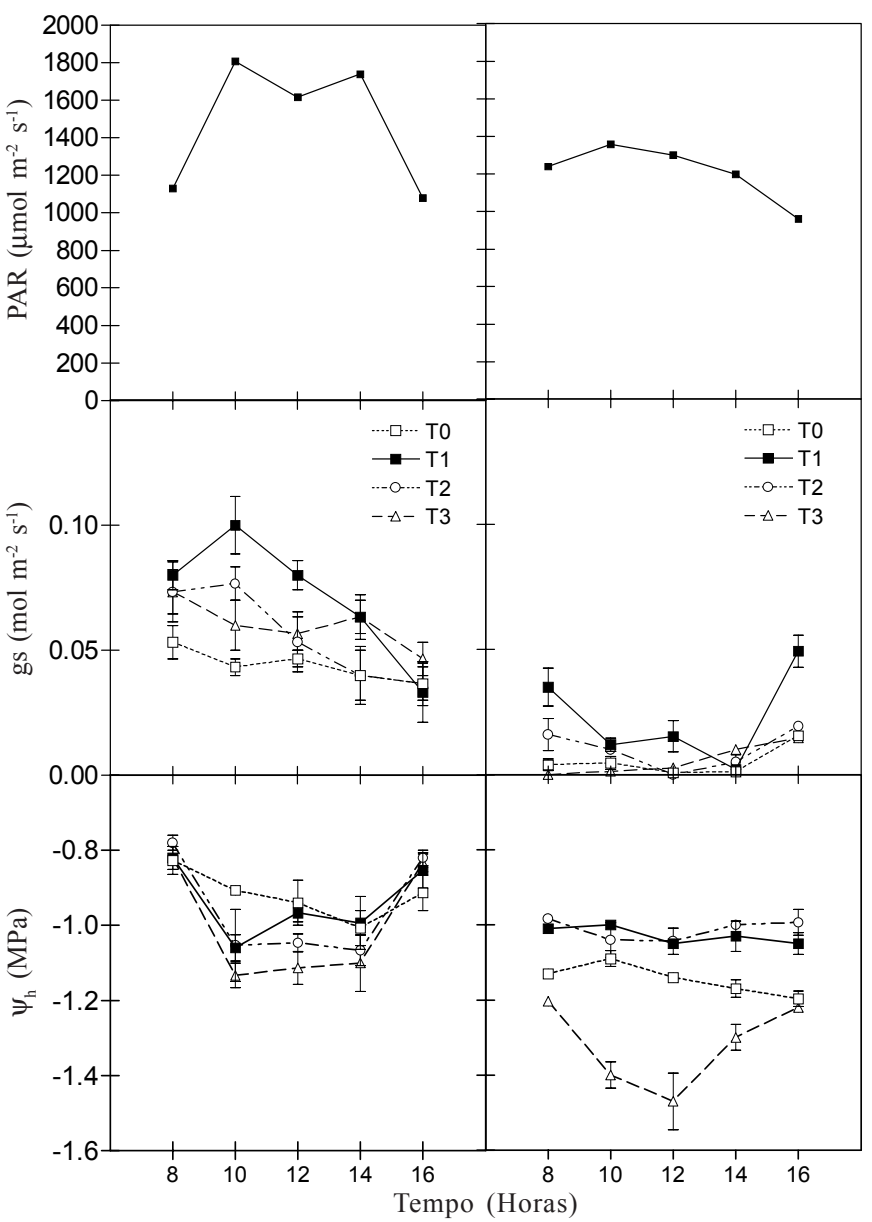

Figura 6. Curso diário da radiação fotossintética ativa (PAR), condutância estomática (gs) e do potencial hídrico foliar $\left(\Psi_{\mathrm{h}}\right)$ em plantas do coqueiro "Gigante do Brasil", sob diferentes tratamentos. A - Período chuvoso (Agosto/92) e B - Período $\operatorname{seco}\left(\right.$ Março/93). $T_{0}$ - testemunha, $\mathrm{T}_{1}$ - aplicação de água doce, $\mathrm{T}_{2}$ e $\mathrm{T}_{3}$ - aplicação de água contendo 7,5 e $15 \mathrm{~g} \mathrm{~L}^{-1}$ de sais totais, respectivamente volume de água aplicada também contribuiu para o maior efeito dos sais no potencial hídrico foliar.

Durante a estação das chuvas (Fig. 6A), a condutância estomática (gs) das plantas irrigadas com água doce foi superior à dos demais tratamentos, apresentando diferença estatística comparada ao tratamento $\mathrm{T}_{0}(\mathrm{p}<0,05)$; no entanto, na estação seca a condutância estomática para todos os tratamentos foi reduzida na ordem de $50 \%$. Após a aplicação da água, às $15 \mathrm{~h}$, ficou mais acentuada a recuperação da abertura estomática do tratamento $\mathrm{T}_{1}$. Ficou evidente, também, que o déficit hídrico e o acúmulo de sais no solo proporcionaram sérias restrições quanto ao comportamento estomático em todos os tratamentos, apesar do elevado valor da radiação fotossinteticamente ativa (PAR).

A redução da condutância estomática foi também detectada por Voleti et al. (1993) em três diferentes genótipos de coqueiro em condições de campo, quando os valores do potencial hídrico foliar eram de aproximadamente -1,4 MPa. Esses autores encontraram redução da condutância de 55 a $230 \%$ na estação seca. Os resultados obtidos por Chartzoulaskis \& Klapaki (2000) em pimenta e, no tomate, por Romero-Aranda et al. (2001), sob condições controladas, mostram que o aumento da concentração em sais induziu a redução da condutância estomática. Pomier \& Brunin (1974) constataram que plantas de coqueiro, quando irrigadas com água salobra contendo $15 \mathrm{~g} \mathrm{~L}^{-1}$, em condições de campo, tiveram a abertura estomática reduzida, quando comparada com plantas irrigadas com água doce.

\section{CONCLUSÕES}

1. O coqueiro, quando recebe irrigação com água salina, apresenta mecanismos para evitar o estresse hídrico, com redução da área foliar e fechamento dos estômatos.

2. Caso não seja disponível água de boa qualidade, a aplicação de água salina durante os meses de déficit hídrico, em solo arenoso, pode ser um meio de se evitar perda de plantas durante as fases jovem e adulta.

\section{LITERATURA CITADA}

Alarcón, J.J.; Sánchez-Blanco, M.J.; Bolarín, M.C.; Torrecillas, A. Water relations and osmotic adjustment in Lycopersicon esculentum and $L$. pennellii during short-term salt exposure and recovery. Physiologia Plantarum, Copenhagen, v.89, p.441-447, 1993.

Alexander, D.; Woodham, R.C. Relative tolerance of rooted cuttings of four Vinifera varieties to sodium chloride. Australian Journal of Experimental Agriculture and Animal Husbandry, Collingwood Victoria, v.44, p.461-465, 1968.

Bernstein, N.; Läuchli, A.; Silk, W.K. Kinematics and dynamics of sorghum (Sorghum bicolor L.). Leaf development at various $\mathrm{Na} / \mathrm{Ca}$ salinities. I. Elongation growth. Plant Physiology, Rockville, v.103, p.1107-1114, 1993a.

Bernstein, N.; Silk, W. K.; Läuchli, A. Growth and development of sorghum leaves under conditions of $\mathrm{NaCl}$ stress. Spatial and temporal aspects of leaf growth inhibition. Planta, New York, v.191, p.433-439, 1993b. 
Bourgeais-Chaillou, P.; Perez-Alfocea, F.; Guerrier, G. Evolution ontogénique de la tolérance au $\mathrm{NaCl}$ chez le soja: Comparaison des réponses au sel à deux stades de développement et chez les cals correspondants. Canadian Journal of Botany, Ottawa, v.70, p.1346-1354, 1992.

Boutelier, E Essai du chlorure de sodium sur la physiologie du cotonnier, Gossypium hirsutum, son rôle dans l'acquisition de la résistance à la sécheresse. Paris: Université Paris VI, 1986. Thèse Doctorat

Chartzoulaskis, K.; Klapaki, G. Response of two greenhouse pepper hybrids to $\mathrm{NaCl}$ salinity during different growth stages. Scientia Horticulturae, Amsterdam, v.86, p.247-260, 2000.

Coudret, A. Action du $\mathrm{NaCl}$ sur les contraintes et les relations hydriques dans les parties aériennes de Plantago maritima et Plantago lanceolata. Oecologia Plantarum, Paris, v.2, n.16, p.111-120, 1981.

Coudret, A.; Louguet, P. Etude comparée de l'action de $\mathrm{NaCl}$ sur les mouvements stomatiques de Plantago maritima L. Var. Gramineae et de Plantago lanceolata L. Physiologie Végétal, Paris, v.18, n.1, p.55-68, 1980.

Dudal, R.; Purnell, M.F. Land resource: Salt affected soil. In: Lennard, E.G.B.; Malcolm, C.V; Stern, W.S.; Wilkins, S.M. Forage and fuel production from salt affected wasteland. Proceedings of Research for Development Seminar, 19-27 May 1984, at Cunderdin, Western Australia. Amsterdam: Elsevier, 1986. 459p.

Ghassemi, F.; Jakeman, A.J.; Nix, H.A. Salinisation of land and water resources: Human causes, extent, management and case studies. Sydney: University New South Wales Press. 1995. 526p.

Greenway, H.; Munns, R. Mechanisms of salt tolerance in nonhalophytes. Annual Review of Plant Physiology, Palo Alto, v.31,p.149-190, 1980.

Grunberg, K.; Taleisnik, E. Salt tolerance in tomato: An assessment of the contribution of phloem retranslocation to the sodium balance of growing leaves. Plant Physiology and Biochemistry, Paris, v.29, n.6, p.559-564, 1991.

Hargreaves, H.H. Potential evapotranspiration and irrigation requeriments for Northeast of Brazil. Logan: Utah State University. 1974. 66p.

Katerji, N.; van Hoorn, J.W.; Hamdy, A.; Mastrorilli, T.; Erskine, W. Response of two varieties of lentil to soil salinity. Agriculture Water Management, Amsterdam, v.47, p.179-190, 2001.
Lin, G.; Sternberg, L. da S.L. Effects of salinity fluctuations on photosynthetic gas exchange and plant growth of the red mangrove (Rhizophora mangle L.). Journal of Experimental Botany, Oxford, v.44, p.9-16, 1993.

Malavolta, E.; Vitti, G.C.; Oliveira, S.A. Avaliação do estado nutricional das plantas: Princípios e aplicações. Piracicaba: Associação Brasileira para Pesquisa da Potassa e do Fosfato, 1989. 201p.

Mennen, H.; Jacoby, B.; Marschnner, H. Is sodium proton antiport ubiquitous in plant cell? Journal of Plant Physiology, Rockville, v.137, p.180-183, 1990.

Munns, R.; Termaat, A. Whole-plant response to salinity. Australian Journal of Plant Physiology, Collingwood, v.13, p.143-160, 1986.

Pomier, M.; Brunin, C. Irrigation des cocotiers à 1'eau sallée. Oléagineux, Paris, v.29, n.4, p183-186, 1974.

Remison, S.U.; Iremiren, G.O. Effect of salinity on the performance of coconut seedling in two contrasting soils. Cocos, Sri lanka, v.8, p.33-39, 1990.

Romero-Aranda, R.; Soria, T.; Cuartero, J. Tomato plant-water uptake and plant-water relationships under saline growth conditions. Plant Science, Ireland, v.160, p.265-272, 2001.

Rozema J.; van Diggelan, J. A comparative study of growth and photosynthesis of four halophytes in response to salinity. Acta Oecologia, Paris, v.12, n.5, p.673-681, 1991.

Scholander, P.F.; Hammel, H.T.; Bradstret, E.D.; Hemmingsen, E.A. Sap pressure in vascular plants. Science, Cambridge, v.148, p.339-346, 1965.

Sobral, L.F.; Santos, Z.G. dos. Sistemas de recomendações de fertilizantes para o coqueiral (Cocos nucifera L.) com base na análise foliar. Aracaju: Embrapa-CNPCo, 1987. 23p. Embrapa-CNPCo. Documentos, 7

Voleti, S.R.; Kasturibal, K.V.; Rajagopal, V.; Nambiar, C.K.B. Influence of soil type on the development of moisture stress in coconut (Cocos nucifera L.) genotypes. Oleagineux, Paris, v.48, p.505-509, 1993.

Ziska, L.H.; Hutmacher, R.B.; Hoffman, G.J.; Jong, T.M. de. Changes in leaf water status associated with salinity in mature field grown Prunus salicina. Physiologia Plantarum, Copenhagen, v.77, p.141-149, 1989. 